\title{
Effects of Surface Modification of MWCNT on the Mechanical and Electrical Properties of Fluoro Elastomer/MWCNT Nanocomposites
}

\author{
Tao $\mathrm{Xu}^{1}$ and Jinghui Yang ${ }^{2}$ \\ ${ }^{1}$ Institute of Chemical Materials, Chinese Academy of Engineering Physics, Mianyang 621900, China \\ ${ }^{2}$ Department of Polymer Materials and State Key Laboratory of Polymer Materials Engineering, Sichuan University, \\ Chengdu 610065, China
}

Correspondence should be addressed to Tao Xu, xuzhtao@126.com

Received 11 October 2011; Accepted 13 January 2012

Academic Editor: Sulin Zhang

Copyright ( $\odot 2012$ T. Xu and J. Yang. This is an open access article distributed under the Creative Commons Attribution License, which permits unrestricted use, distribution, and reproduction in any medium, provided the original work is properly cited.

\begin{abstract}
Surface modification is a good way to improve the surface activity and interfacial strength of multiwalled carbon nanotubes (MWCNTs) when used as fillers in the polymer composites. Among the reported methods for nanotube modification, mixed acid oxidation and plasma treatment is often used by introducing polar groups to the sidewall of MWCNT successfully. The purpose of this study is to evaluate the effect of different surface modification of MWCNT on the mechanical property and electrical conductivity of Fluoro-elastomer (FE)/MWCNT nanocomposites. MWCNTs were surface modified by mixed oxidation and $\mathrm{CF}_{4}$ plasma treatment and then used to reinforce the fluoro elastomer (FE, a copolymer of trifluorochloroethylene and polyvinylidene fluoride). FE/MWCNT composite films were prepared from mixture solutions of ethylacetate and butylacetate, using untreated CNTs (UCNTs), acid-modified CNTs (ACNTs), and $\mathrm{CF}_{4}$ plasma-modified CNT (FCNTs). In each case, MWCNT content was $0.01 \mathrm{wt} \%$, $0.05 \mathrm{wt} \%, 0.1 \mathrm{wt} \%$, and $0.2 \mathrm{wt} \%$ with respect to the polymer. Morphology and mechanical properties were characterized by using scanning electron microscopy (SEM), Raman spectroscopy, as well as dynamic mechanical tests. The SEM results indicated that dispersion of ACNTs and especially FCNTs in FE was better than that of UCNTs. DMA indicated mechanical properties of FCNT composites were improved over ACNT and UCNT filled FE. The resulting electrical properties of the composites ranged from dielectric behavior to bulk conductivities of $10^{-2} \mathrm{Sm}^{-1}$ and were found to depend strongly on the surface modification methods of MWCNTs.
\end{abstract}

\section{Introduction}

CNT/polymer nanocomposites hold the promise of delivering exceptional mechanical properties and multifunctional characteristics $[1,2]$, however, the scope of CNT applications in practical devices has been largely hampered by their poor dispersibility in polymer resin and weak interfacial bonding with polymer matrix. Ineffective interfacial bonding and sliding of individual nanotubes within the ropes inhibit load transfer from the matrix to the fillers in the composite, making the amount of mechanical reinforcement unachievable in polymer composites [3-6]. Therefore, surface modification of carbon nanotubes is a useful approach to improve their surface activity and processibility. Moreover, modification introduces suitable covalent bonds on the sidewalls of $\mathrm{CNTs}$, by which the chemical bondings in the interface between the CNT and the polymer matrix can be obtained [7-9].

Reported methods for nanotubes modification include chemical and physical treatments [10-14]. Among these methods, mixed acid oxidation and the RF-plasma treatment is often used because both of them can successfully graft polar groups on CNTs [15-20]. For mixed acid oxidation, hydroxyl and carbonyl groups are reported to be grafted on the sidewalls of CNTs. On the other hand, many scientists have reported that the surface of CNTs film can be fluorinated after exposure to $\mathrm{CF}_{4}$ plasma. The relative amount of functional groups could be controlled by parameter adjustment during $\mathrm{CF}_{4}$ plasma treatment. Besides semi-ionic/covalent bonding on the surface [21], polymerization of a fluoropolymer around the CNT surface could be also obtained, depending on the reaction conditions. Our previous work $[22,23]$ 
has provided a new way for the homogeneous fluorination of MWCNT powder by using homemade plasma equipment.

As we all know, fluoro-elastomer is quite difficult to be reinforced due to its bad compatibility with the filler and high spatial shielding effect. The fluoro-elastomer we used in the research is a copolymer of trifluorochloroethylene and polyvinylidene fluoride with the volume ratio $1: 1$, which is one of the important materials employed in the aerospace and automotive industries. However, its application is limited by the relatively lower strength. In order to achieve optimal enhancement in the mechanical properties of FE/CNTs nanocomposites, two key issues should be considered: homogeneous dispersion of CNTs in the fluoro-elastomer and strong interfacial bonding between CNT and FE matrix [24]. In order to accomplish these two tasks, surface modification of CNTs is an effective way. But how to select suitable surface modification method is still a challenge for polymer scientists. In this paper, we report on our attempts to understand the relationship between the surface modification of carbon nanotubes and the resulting mechanical property and the electrical conductivity of the nanocomposites. FE/CNTs nanocomposites were fabricated by wet-casting with different kinds of carbon nanotubes including untreated, acid treated, and $\mathrm{CF}_{4}$ plasma treated. It is shown that fluorinated carbon nanotubes can affect the dispersion in FE rubber and also lead to enhancement behavior on mechanical properties of FE/CNTs composites. In addition, the specific bulk conductivity of the materials was analyzed by DC impedance spectroscopy, and the results showed that the final electrical network depended on the surface modification of CNTs and its weight volume in the matrix. CNTs treated by mixed acid can improve the electrical conductivity of FE/ACNTs with lower percolation threshold. The FE/CNTs composites with improved strength and electrical conductivity are expected to be applied in the fields of automotive and aerospace industry.

\section{Experiment}

\subsection{Samples and Its Modification}

2.1.1. Samples. MWCNTs prepared by chemical vapor deposition (CVD) were purchased from the Organic Chemical Limited Company, Chengdu, China. The lengths were about $50 \mu \mathrm{m}$, and the outer diameters ranged from 60 to $80 \mathrm{~nm}$. Fluoro-elastomer is the copolymer of trifluorocholorethylene and polyvinylidene fluoride with the malor ratio $1: 1$, which was purchased from the Chenguang Company, Zigong, China.

2.1.2. Mixed Acid Treatment. A raw-MWCNTs sample (4.0 g) was mixed with the concentrated $\mathrm{H}_{2} \mathrm{SO}_{4}(98 \%)$ and $\mathrm{HNO}_{3}$ (65\%) mixed solution ( $3: 1$ by volume) for $24 \mathrm{~h}$ at room temperature with stirring. Finally, the solution was filtered through a cellulose nitrate filter (pore size $\sim 0.2 \mathrm{~mm}$ ) and dried at $60^{\circ} \mathrm{C}$ in a vacuum oven for $24 \mathrm{~h}$. The as-prepared MWCNTs $(2.5 \sim 3.0 \mathrm{~g})$ with a yield of approximately $60 \sim 70 \%$ were obtained.
2.1.3. Plasma Treatment. Before being modified by $\mathrm{CF}_{4}$ plasma, the as-received MWCNT was cleaned by a classical wet method using nitric acid in order to remove the metal catalysts. The inductive coupled plasma was generated in the radio frequency plasma modification equipment (RF-600, Southwest Academy of Nuclear Physics) with a rotating barrel fixed between the two discharge electrodes. A controlled flow of $\mathrm{CF}_{4}$ gas was introduced into the chamber. The reflective frequency was $13.56 \mathrm{MHz}$. The diameter of radio-frequency plate electrode was approximately $350 \mathrm{~mm}$, and the spacing of the electrode and samples was $150 \mathrm{~mm}$. The $\mathrm{CF}_{4}$ plasma treatment conditions for MWCNT powder were as follows: gas flow rate of $80 \mathrm{sccm}$, operating pressure of $10 \mathrm{~Pa}$, a bias of $200 \mathrm{~V}$, power of $300 \mathrm{~W}$, process duration of $10 \mathrm{~min}-$ utes, and the average temperature of samples at about $100^{\circ} \mathrm{C}$ during the $\mathrm{CF}_{4}$ plasma treatment. Details were presented in the previous papers $[22,23]$. The untreated, acid-treated, and plasma-treated carbon nanotubes were abbreviated as CNTs, ACNTs, and FCNTs. And the scheme of modification and mixing in $\mathrm{FE}$ is shown in Figure 1.

2.2. Preparation of the FE/CNT Composites. The fabrication of FE/MWCNTs composites is based on a convenient solution process. In brief, FE was first dissolved in the mixture of ethyl acetate and butyl acetate for 2 days till the uniform solution formed. The exact amount of MWCNTs was dissolved in ethyl acetate with continuous ultrasonication for 30 minutes. Then the FE solution was added to the MWCNT solution to obtain a MWCNT-to-polymer weight ratio of $0.05-0.2 \mathrm{wt} . \%$ while stirring continually. The solution was then sonicated for 5 min using a high-power sonic tip $(200 \mathrm{~W})$ followed by a mild sonication for $2 \mathrm{~h}$ in a sonic bath. After careful mixing of FE solution with carbon nanotubes followed by subsequently casting and controlled solvent evaporation, freestanding FE/MWCNTs composite films were obtained by peeling off from Teflon disks. For the control sample, pure FE films were obtained under the same fabrication processing.

\subsection{Characterization}

2.3.1. X-Ray Photoelectron Spectroscopy (XPS). In order to determine the surface chemical changes during the treatments, XPS measurements were used with a hemispherical electron energy analyzer (ESCALAB250, England). A monochromatized $\mathrm{Al} \mathrm{K} \alpha$ line $(h v=1486.6 \mathrm{eV})$ was used as the photon source, and the photoelectrons were collected at an angle of $55^{\circ}$ relative to the sample surface normal. The energy resolution of system was $0.9 \mathrm{eV}$. In the spectrum analysis, the background signal was subtracted by Shirley's method. The samples were prepared by attaching carbon nanotube powders to a scotch conductive tape in a form of thin film, in order to avoid dispersion during the pumping. The software Avantage 2.52 was used for peak fitting and quantitative anal ysis.

2.3.2. Zeta-Potential Measurement. Zeta-potential of different kinds of CNTs was measured by Malvem nanoparticles 


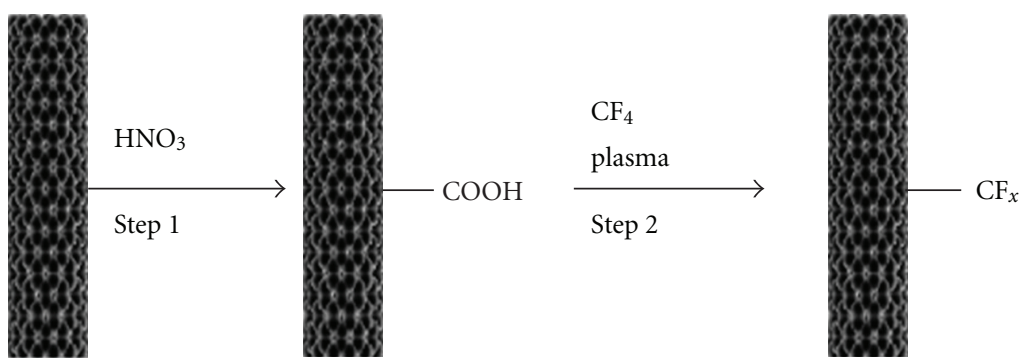

(a)
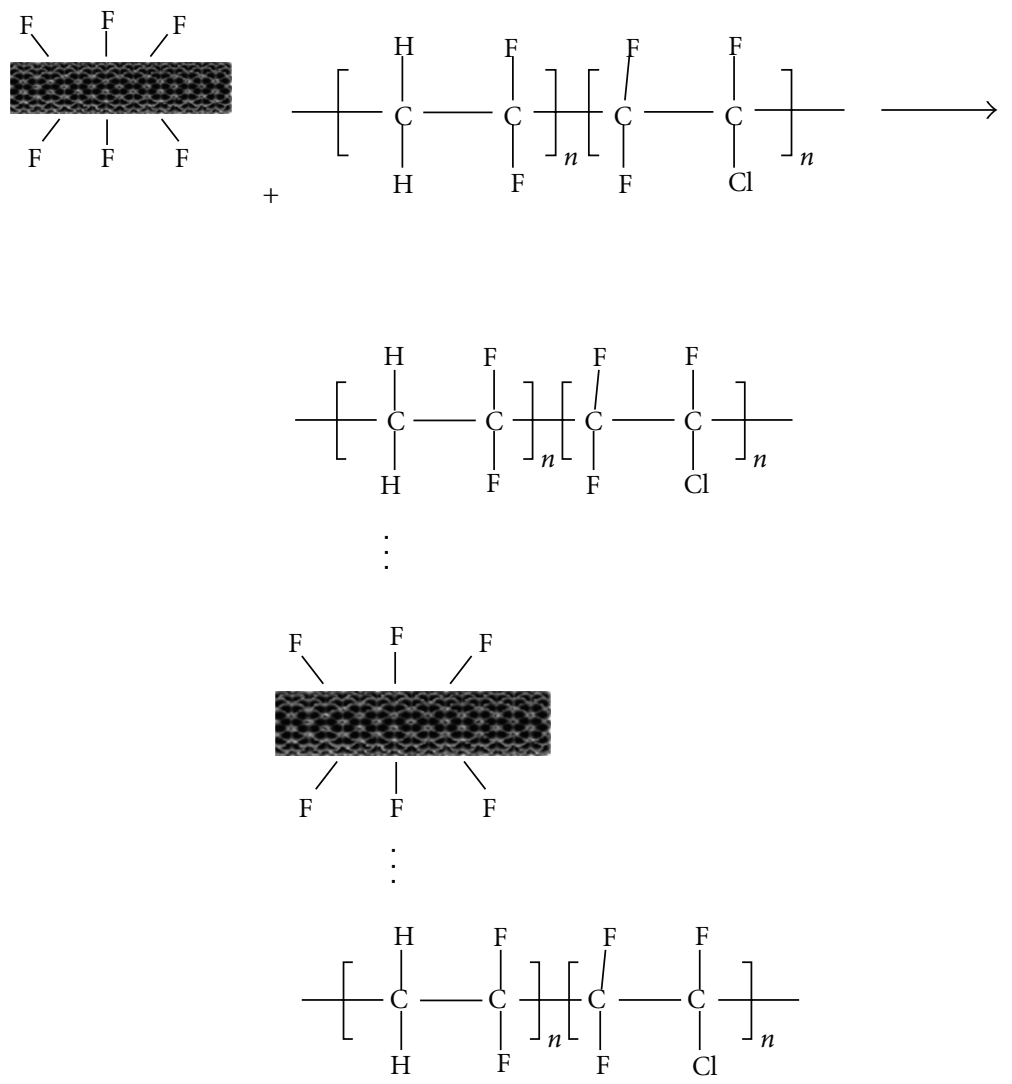

(b)

FIGURE 1: Scheme of surface modification and mixing: (a) $\mathrm{CF}_{4}$ plasma modification of MWCNT; (b) suggested hydrogen bonding of FE with FCNT.

analyzer. CNTs were dispersed in distilled water at the same percent $(0.1 \%)$ and vibrated for 10 minutes.

2.3.3. Scanning Electron Microscopy (SEM). Samples were fractured in liquid nitrogen, and the fracture surface was observed under an acceleration voltage of $20 \mathrm{kV}$ with a JEOL JSM-5900LV for SEM experiment.

2.3.4. Micro-Raman Spectroscopy. Micro-Raman spectra were recorded on a Renishaw system 2000 micro-Raman spectrometer with Ar (514 nm wavelength) as excitation source. The incident light was introduced to the sample through a $50 \mathrm{X}$ objective as a spot less than $2 \mu \mathrm{m}$ in diameter with the power no more than $1 \mathrm{~mW}$. Three different spots were measured and overlapped.
2.3.5. Mechanical Property. The investigation of the thermomechanical behavior was performed by dynamic-mechanical thermal analysis, DMA, using TA RSA3 8500-0001 system. For the measurements, rectangular specimens of $50 \mathrm{~mm}$ length, $5 \mathrm{~mm}$ width, and $2 \mathrm{~mm}$ thickness were prepared. The tests were performed in tensile mode at a frequency of $10 \mathrm{~Hz}$ with a static strain of $0.6 \%$ and a dynamic strain of $0.1 \%$, in a temperature range between $-20^{\circ} \mathrm{C}$ and $70^{\circ} \mathrm{C}$ with a heating rate of $2^{\circ} \mathrm{C} / \mathrm{min}$.

2.3.6. Electrical Conductivity. DC conductivity was measured with a Keithley 6514 Digital Electrostatic Charge Meter in a four-probe setup at room temperature and reported as an average of three readings (see Table 1). The whole measurements were operated under high vacuum conditions in preventing environmental influence. 
TABLE 1: Experimental values of the conductivity for FE/CNT nanocomposites.

\begin{tabular}{lccc}
\hline Sample & Film thickness, $\mu \mathrm{m}$ & Resistivity, $\Omega \cdot \mathrm{m}$ & Conductivity, S/m \\
\hline FE/CNT0.01 & 102.0 & - & - \\
FE/CNT0.05 & 13.1 & $1.13 \times 10^{8}$ & $8.8 \times 10^{-9}$ \\
FE/CNT0.1 & 73.0 & $1.27 \times 10^{7}$ & $8.1 \times 10^{-8}$ \\
FE/CNT0.2 & 69.6 & $4.03 \times 10^{2}$ & $2.5 \times 10^{-3}$ \\
\hline FE/ACNT0.01 & 82.7 & $3.58 \times 10^{9}$ & $2.8 \times 10^{-10}$ \\
FE/ACNT0.05 & 44.0 & $4.18 \times 10^{3}$ & $2.4 \times 10^{-4}$ \\
FE/ACNT0.1 & 30.0 & $7.96 \times 10^{2}$ & $1.2 \times 10^{-3}$ \\
FE/ACNT0.2 & 56.3 & 26.9 & $3.7 \times 10^{-2}$ \\
\hline FE/FCNT0.01 & 54.0 & $4.47 \times 10^{9}$ & $2.2 \times 10^{-10}$ \\
FE/FCNT0.05 & 100.0 & $4.66 \times 10^{8}$ & $2.1 \times 10^{-9}$ \\
FE/FCNT0.1 & 210.0 & 33.8 & $2.9 \times 10^{-4}$ \\
FE/FCNT0.2 & 180.0 & 28.3 & $3.5 \times 10^{-3}$ \\
\hline
\end{tabular}

\section{Results and Discussion}

First, the effects of surface modification on the chemical states and morphology of the MWCNTs were estimated. XPS C1s spectrum of untreated MWCNTs has three chemical states, as shown in Figure 2. The main peak (1) at $283.8 \mathrm{eV}$ corresponds to the $\mathrm{sp}^{2}$ carbon atoms of original carbon nanotube, similar to the graphite; (2) $284.7 \mathrm{eV}$ matches with the $\mathrm{sp}^{3}$ carbon atoms indicates the amorphous carbon layer at the surface of CNTs; (3) $289.0 \mathrm{eV}$ is attributed to the oxygen-carbon from air contamination $[17,18]$. This is consistent with the fact that nonpurified nanotube powder also contains amorphous carbon and that defects exist at the surface of the nanotubes $[5,14]$. After being modified by mixed acid, the CNT main structure survived the irradiation as the chemical state of $\mathrm{sp}^{2}$ carbon at $283.9 \mathrm{eV}$ remains the same. In addition, a new peak at $286.4 \mathrm{eV}$ appears and is attributed to oxidation carbon of $\mathrm{C}=\mathrm{O}$. After being modified by $\mathrm{CF}_{4}$ plasma, more new peaks appear with higher binding energy and are attributed to fluorinated species: $\mathrm{C}-\mathrm{C}, \mathrm{C}-\mathrm{CF}$, $\mathrm{C}-\mathrm{O}, \mathrm{C}-\mathrm{O}-\mathrm{F}$, and $\mathrm{C}-\mathrm{F}$, which indicates that $\mathrm{CF}_{4}$ plasma can induce grafting of $\mathrm{CF}_{x}$ or depositing of a layer of fluorine polymer onto MWCNTs successfully. For a further comparison of different kind of MWCNTs, the SEM is used to observe the morphologies of the carbon nanotube shown in Figure 2 accordingly. The tubes of untreated MWCNTs can be clearly seen, where the amorphous carbon layer is deposited on the surface of CNTs, connecting with each other. No more disordered and amorphous carbon is seen on the surface after mixed acid oxidation. The diameter of the ACNT becomes smaller and the tubes can be more clearly seen. After $\mathrm{CF}_{4}$ plasma treatment, the tubes of MWCNT expand again and the surface becomes rough. A layer of white substrate coated on the surface of MWCNT powder is observed, suggesting a possible formation of fluoropolymer on the MWCNT surface.

In general, the stability of colloidal particles in solution is important for casting process and is greatly affected by their surface charge density. The electrostatic potential of charged particles dispersed in a liquid medium is governed mainly by surface functionality, especially by its ionization ability to produce a charged surface, and the preferential absorption of ions of one charge sign from the solution [25]. Therefore, the zeta potentials of CNTs, ACNTs, and FCNTs were measured to understand the effects of surface charge on the dispersion. As we all know, the higher the absolute value of the zeta potential, the more opposite ions will occur in the diffusion layer and the more stable the suspension will be, which indicate the better dispersion of particles in the solvent. Meanwhile, the poorest quality dispersions occur when the magnitude of the zeta potential of particles is near zero. The lowest value is for UCNTs $(4.62 \mathrm{~V})$, next the ACNTs $(-11.9 \mathrm{~V})$, and the FCNTs have the highest value $(-20.2 \mathrm{~V})$. Obviously, FCNTs exhibit negative shift of zeta potentials compared to the CNTs and ACNTs. And also the negative shift can be attributed to the negative surface charges of FCNTs in the solution. Although the values were measured in the distilled water, the results indicated that the surface charge density of CNTs was changed by surface modification. CNTs with C-F groups grafted may reduce the agglomeration effectively by the addition of electron-attracted groups. Therefore, the addition of $\mathrm{C}-\mathrm{F}$ groups can improve the stability and dispersion of CNTs in the medium, comparing with the pristine CNTs and acid-treated CNTs.

Direct observations of the improved dispersion after adding FCNT are shown in Figure 3, which shows SEM images of the fracture surface of the FE/MWCNT film with MWCNT loading of $0.2 \mathrm{wt} \%$. CNTs without surface modification cannot be dispersed well in the rubber matrix, and most of them are clumped together in aggregates. The dispersion property of ACNT in the FE matrix is a little improved compared with the pristine MWCNT; there are more individual nanotubes scattered in the matrix, while only some of them are clumped together in aggregates in a smaller size. The addition of fluorine functional groups to the CNTs significantly improves the dispersion of the nanotubes in the elastomeric matrix. The different dispersion for three kinds of carbon nanotubes is well consistent with the results of zeta potentials. It is estimated that the addition of containing fluorinated functional group to CNTs surface can effectively 

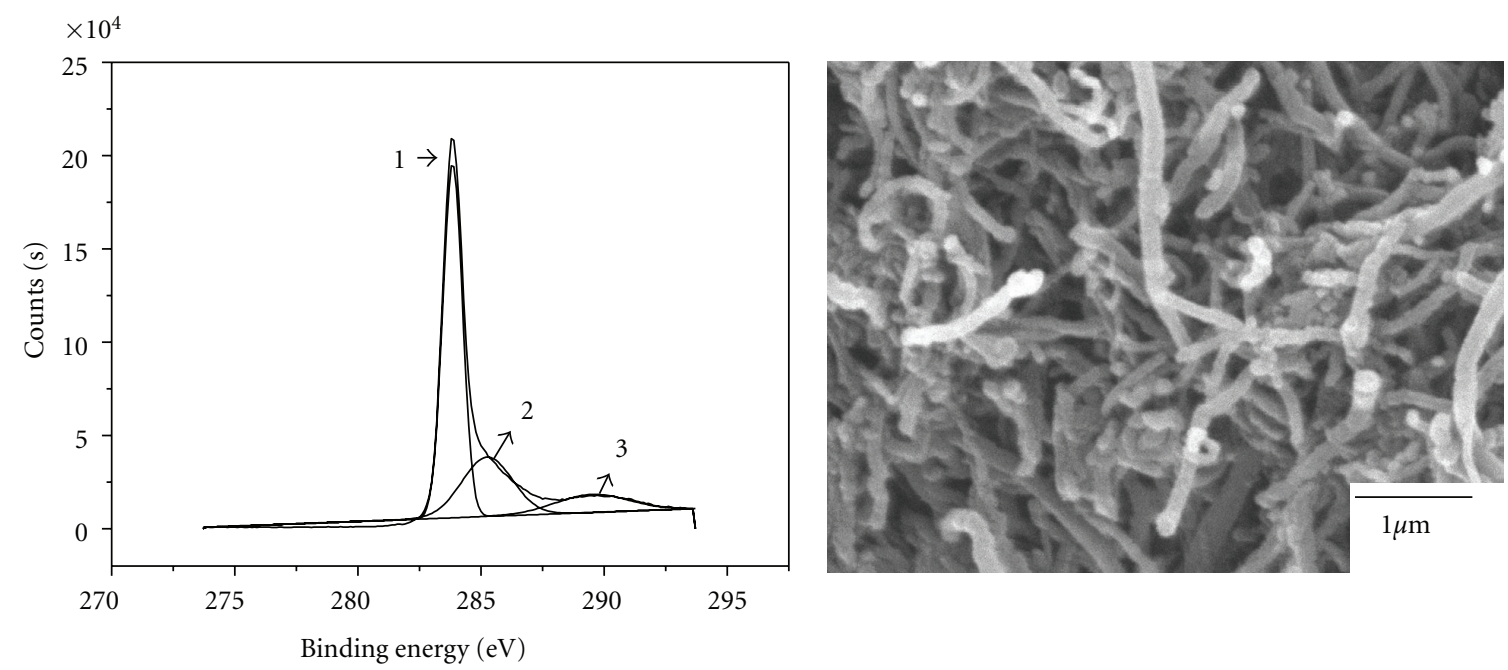

(a)
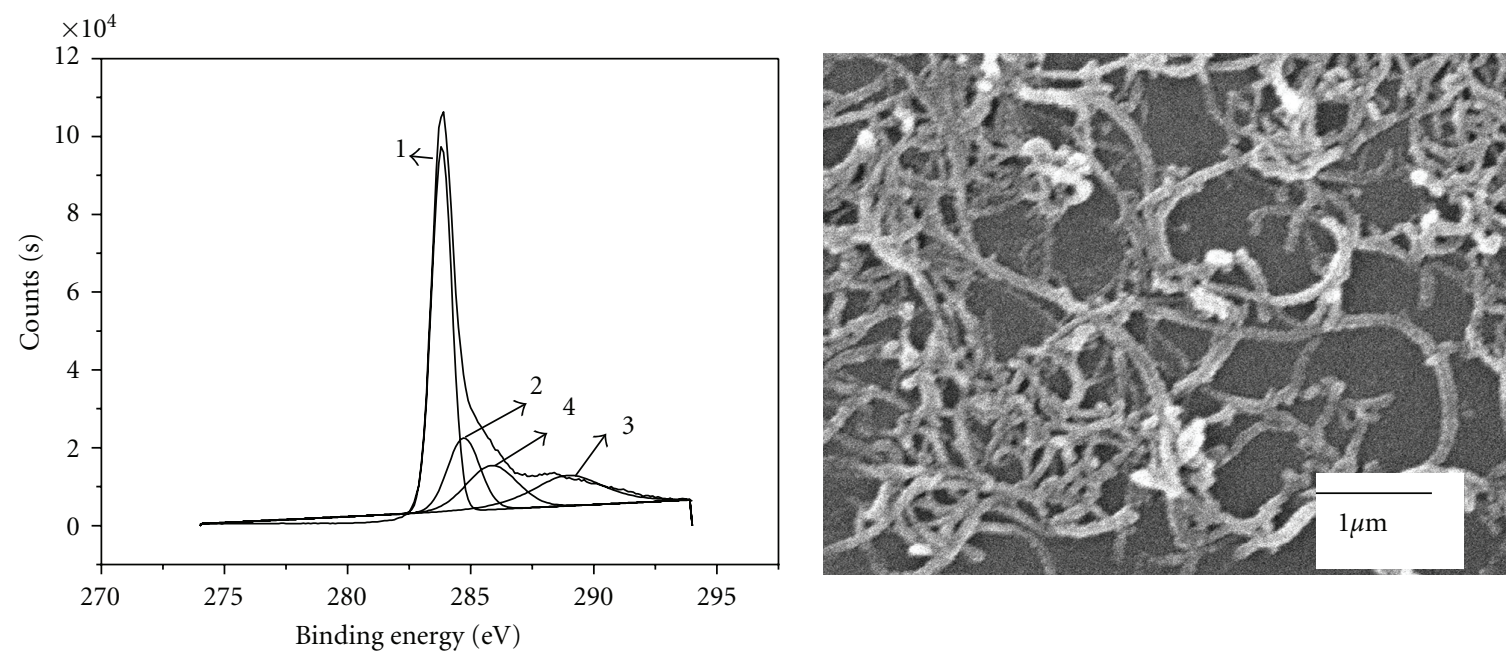

(b)
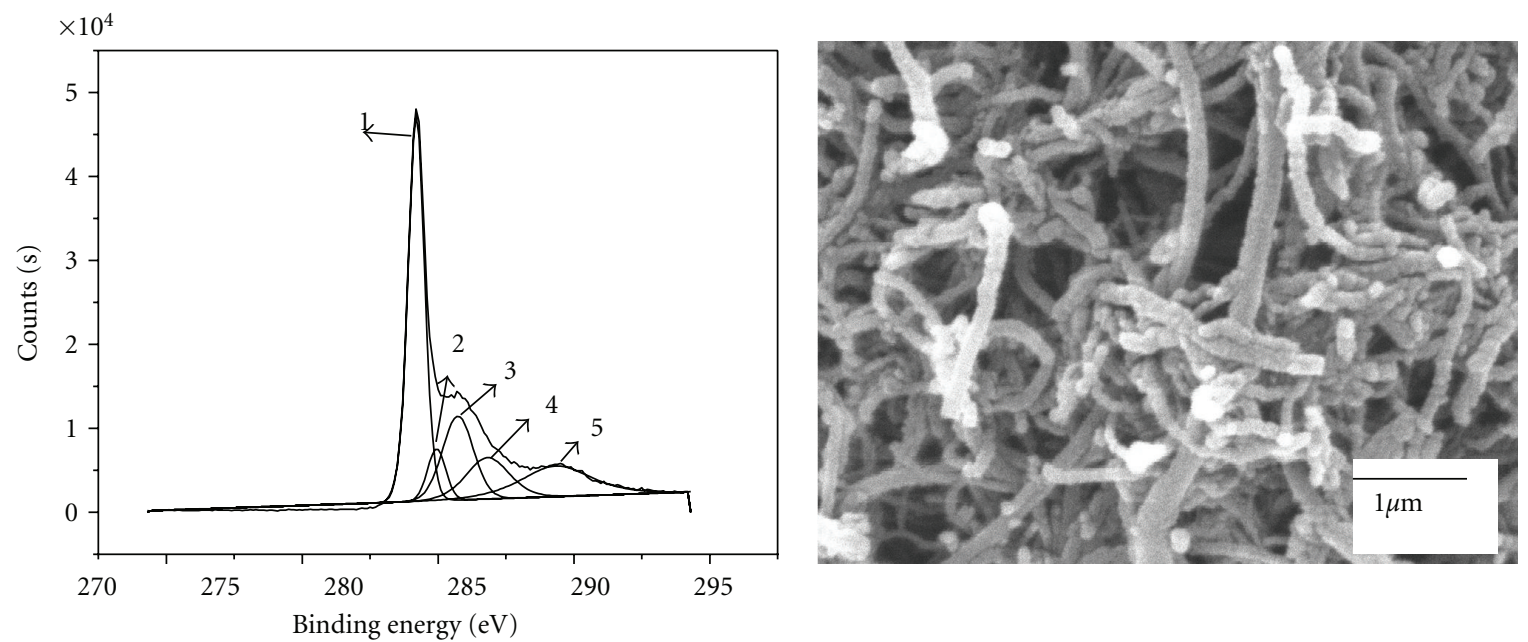

(c)

FIGURE 2: XPS C1s spectra: (a) pristine; (b) acid-oxidation-treated MWCNTs; (c) $\mathrm{CF}_{4}$ plasma-treated MWCNTs. Also shown are the corresponding SEM micrographs. 


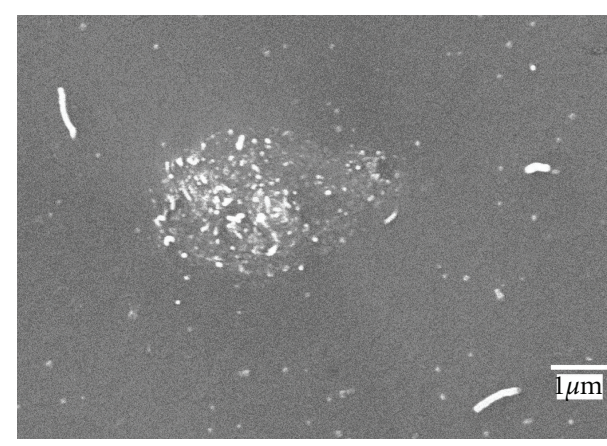

(a)

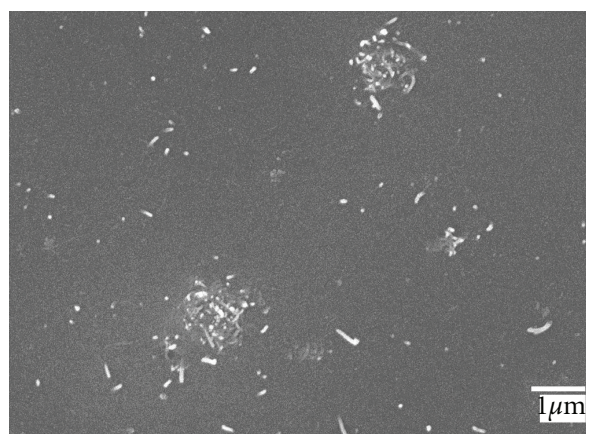

(b)

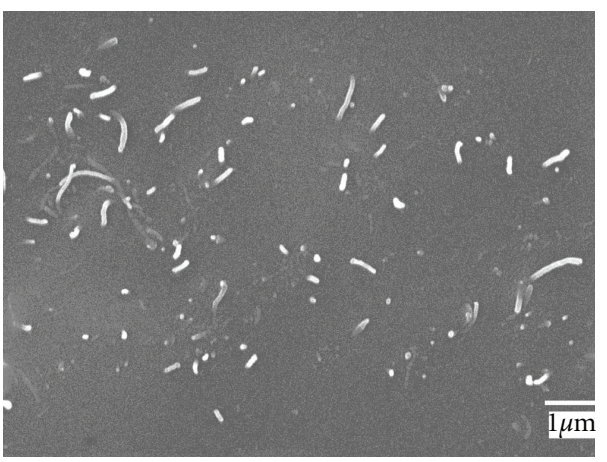

(c)

FIGURE 3: SEM photographs of nanocomposite fracture surfaces showing the dispersion state of MWCNT. (a) $0.2 \mathrm{wt} \%$ UCNT. (b) $0.2 \mathrm{wt} \%$ ACNT. (c) $0.2 \mathrm{wt} \%$ FCNT.

eliminate the Van der Waals force of carbon nanotubes due to the exclusive force of F-F bond, finally resulting in the improved dispersion of CNTs in the solvent and in the FE matrix. Noticeably, seen from Figure 3, the FCNTs are not pulled out from the matrix when samples were prepared for SEM observation, indicating strong interfacial adhesions between FCNT and polymer matrix. The strong interfacial interactions may originate from the better compatibility between fillers and polymer matrix. On the other hand, the hydrogen bonding between FCNT and FE may also contribute to the improved interfacial interaction.

For a further study on the interaction between CNTs and polymer matrix, Raman was used to detect the interaction between CNTs and the FE matrix. From Figure 4, all spectra clearly display peaks at $1352 \mathrm{~cm}^{-1}$ (D line), $1596 \mathrm{~cm}^{-1}$

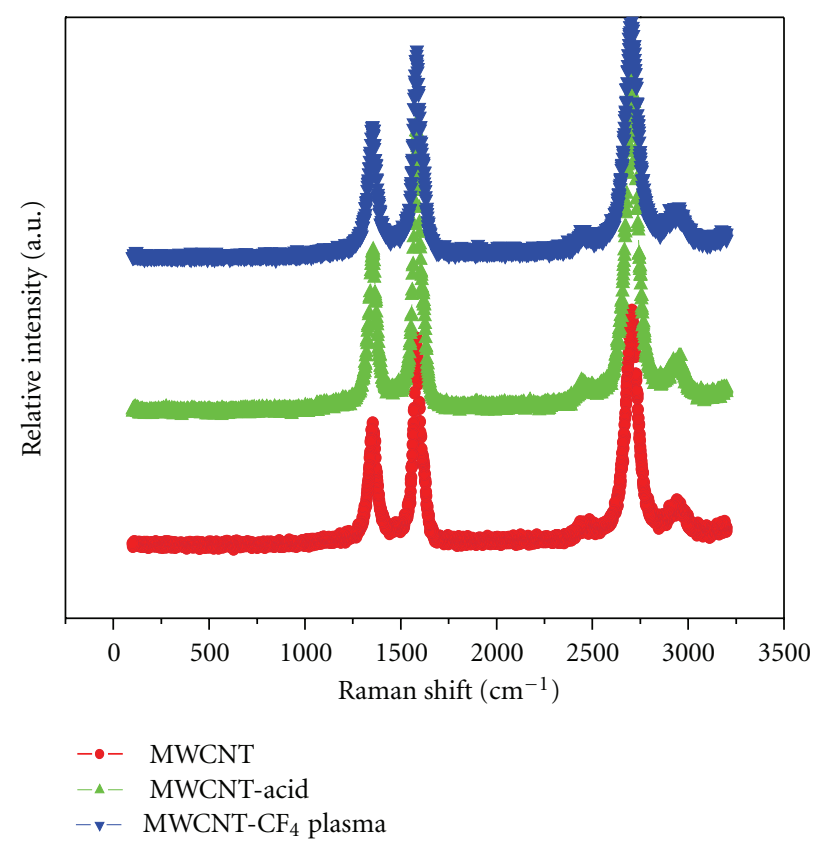

Figure 4: Raman spectra for MWCNT under different treatment.

( $G$ line), and $2650 \mathrm{~cm}^{-1}$ ( $\mathrm{G}^{\prime}$ line). The $\mathrm{D}$ line indicates disordered or amorphous carbons, while the $G$ line indicates graphite or ordered carbons in the MWCNTs, and the $\mathrm{G}^{\prime}$ line indicates a second harmonic of the D line [16]. Evaluating the $\mathrm{D} / \mathrm{G}$ intensity ratio in this Raman spectra, which is frequently used to assess the degree of crystallinity in carbon samples, we can find that a higher ratio exists in the treated MWCNT compared with that of pristine MWCNTs, indicating more defects in the crystal structure $\left(\mathrm{sp}^{3}\right.$ carbons as a result of the formation of carboxyl and hydroxyl groups). Among all kinds of CNT, the FCNT has the highest D/G intensity ratio (0.86), next is the ACNT (0.73), and last is the CNT (0.56). The results indicate that the addition of fluorine to the nanotube sidewalls reduces the intensity of the $\mathrm{sp}^{2} \mathrm{C}=\mathrm{C}$ stretching mode and increases the intensity of the $\mathrm{sp}^{3} \mathrm{C}-\mathrm{C}$ stretching mode. This change is indicative of covalent modification as it reveals $\mathrm{sp}^{3}$ hybridization or disorder within the nanotube framework. Thus the increase in the relative intensity of the D-band can be attributed to an increased number of $\mathrm{sp}^{3}$-hybridizated carbons in the nanotube framework and can be taken as a crude measure of the degree of modification. After mixing and casting process, some of the fluorine is removed from the nanotubes, as evidenced by the return of the $\mathrm{sp}^{2}$ peak at $1596 \mathrm{~cm}^{-1}$ seen in Figure 5. The D/G intensity ratio of FE/FCNT decreased comparing with FE/ACNT and FE/MWCNT composites, which indicates the chemical bonding formed between the FCNT and FE.

The DMA properties were measured for neat FE and nanocomposite films, as shown in Figures 6, 7, and 8. The storage modulus of FE/CNTs is enhanced on increasing the content of CNTs to $0.1 \mathrm{wt} \%$, while a decrease in modulus is observed on further increasing the UCNTs and ACNTs content to $0.2 \mathrm{wt} \%$. The decrease of storage modulus for 


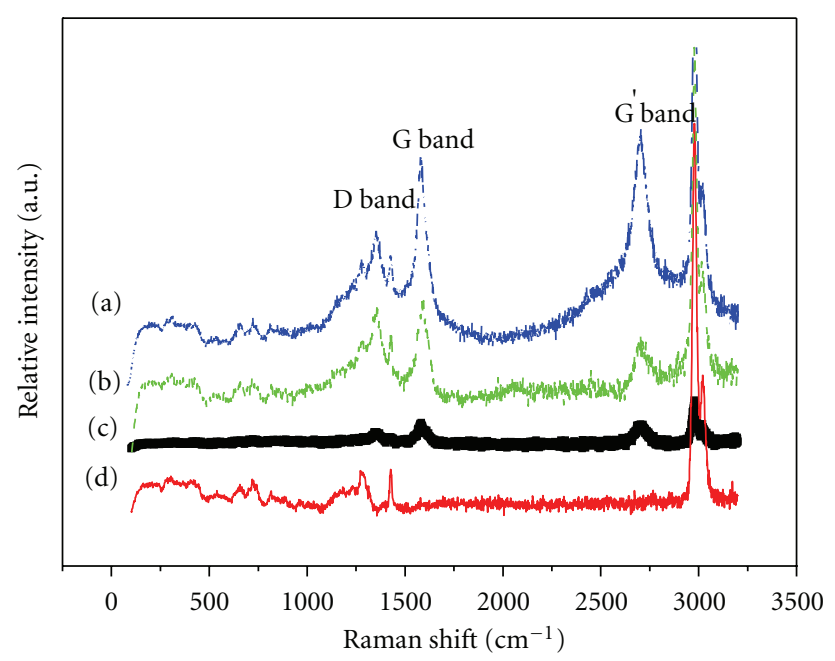

FIgure 5: Micro-Raman spectra of FE and FE/MWCNT nanocomposites: (a) FE/FCNT, (b) FE/ACNT, (c) FE/MWCNT, and (d) FE.

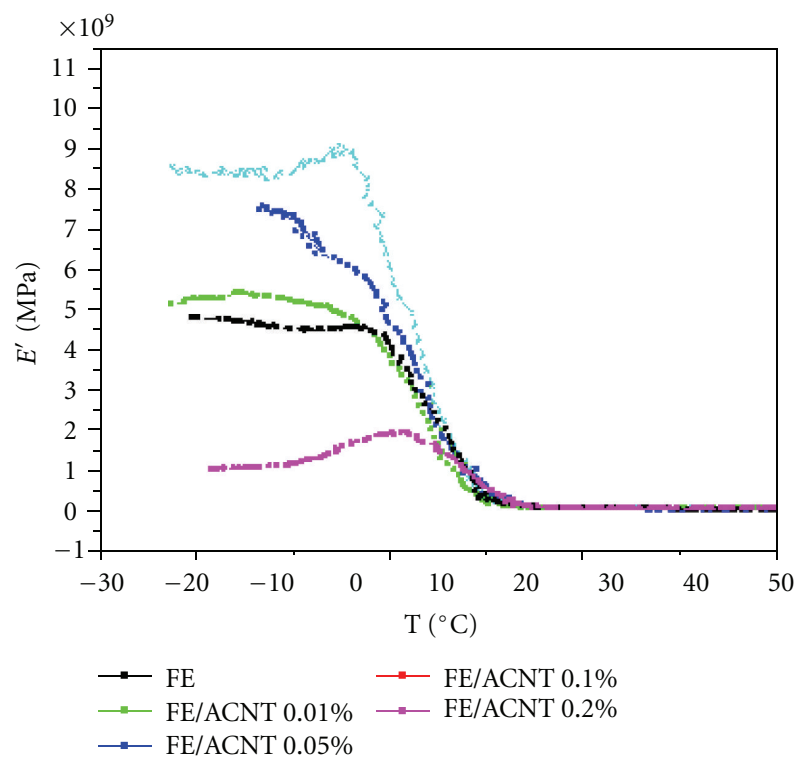

FIGURE 6: The storage modulus as a function of temperature for the FE/MWCNT with different MWCNT loadings.

the nanocomposites with $0.2 \mathrm{wt} \%$ content of UCNTs and $0.2 \mathrm{wt} \%$ ACNTs may be due to the agglomeration in the polymer matrix. On the contrary, the storage modulus for FE/ FCNTs shows a gradual increase of the FCNTs loadings. Even when the loadings of FCNTs increased to $0.2 \mathrm{wt} \%$, the modulus has reached up to maximum as a result of homogeneous dispersion of FCNTs and good interaction between FCNTs and the matrix. Thus the energy dissipation from the matrix to carbon nanotubes due to the strong interaction which is from the compatibility and hydrogen bonding as described above can be achieved, resulting in the increase of storage modulus.

Figure 9 shows the relationship between the conductivity and the weight fraction of MWCNTs with different surface

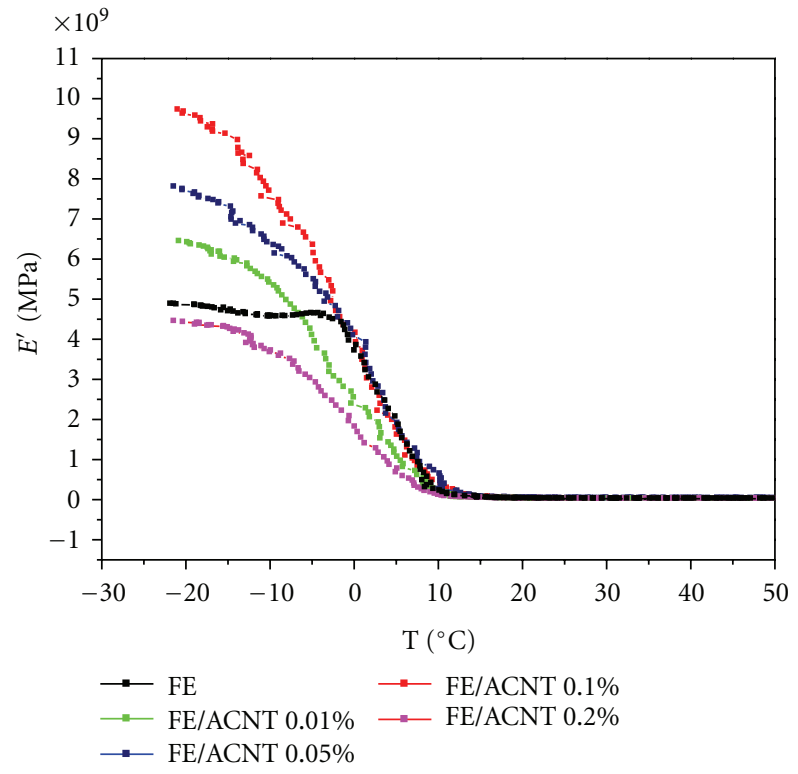

FIgURE 7: The storage modulus as a function of temperature for the FE/ACNT with different ACNT loadings.

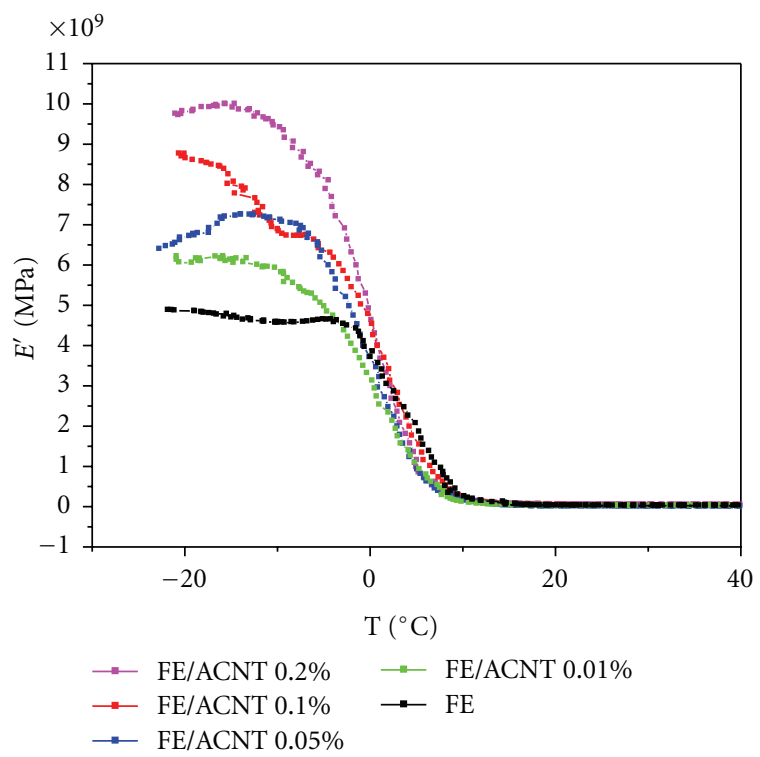

FIGURE 8: The storage modulus as a function of temperature for the FE/FCNT with different FCNT loadings.

modification in the composites. It is obvious that the conductivity of the nanocomposites increases rapidly with increasing MWCNT weight percentage. The electrical properties of the composites ranged from dielectric behavior to bulk conductivities of $10^{-2} \mathrm{Sm}^{-1}$. The increase in $\sigma$ as a function of the MWCNT mass fraction is usually due to the introduction of conducting CNT paths to the polymer, indicative of percolative behavior. Moreover, it was found that the electrical property was dependent strongly on the surface modification methods of MWCNTs. Apparently, the conductivity is the highest for ACNT comparing with FCNT and CNT at 


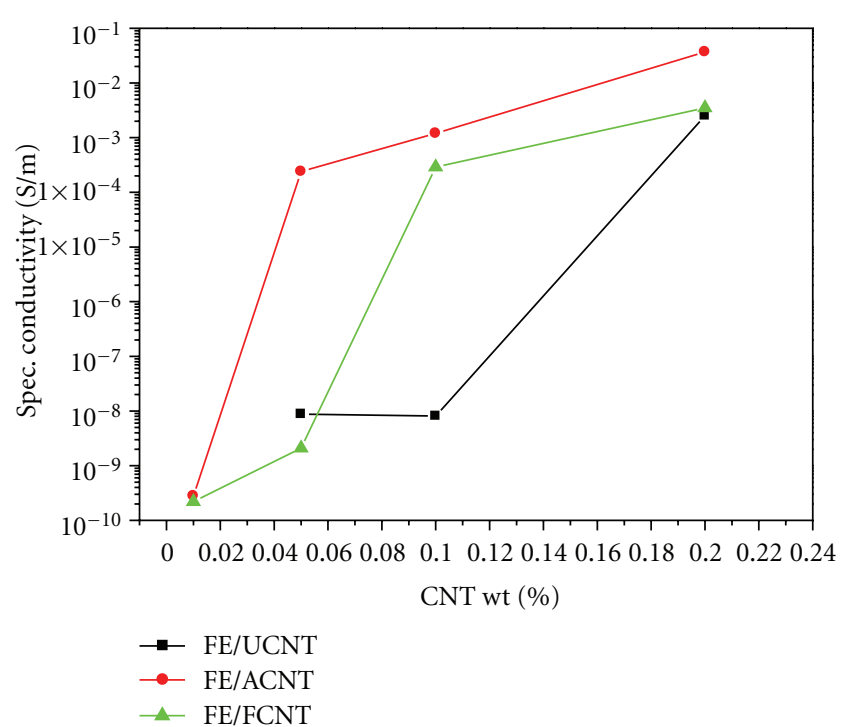

FIGURE 9: The conductivity as a function of CNT loading for CNT/ FE composites.

the same weight loading [26]. It is reported that percolation threshold depended on the shape and aspect ratio of fillers. From SEM micrographs (Figure 2), we can find that CNTs after being acid treated exhibits smooth surface compared with UCNT and FCNT by removing amorphous carbon effectively, which increases the aspect ratio at a given weight percent as well. Therefore, electrical percolation threshold for $\mathrm{FE} / \mathrm{ACNT}$ is the lowest compared with that of FE/CNT and FE/FCNT. On the other hand, there maybe exists a deposited fluoro polymer layer on the surface of $\mathrm{CNT}$ after being $\mathrm{CF}_{4}$ plasma treated, which reduces the electrical conductivity of CNT in the composites. But the conductivity is still higher than that of UCNT, which might be attributed to the improved dispersion state of FCNT in the polymer, and thus reduces percolation threshold of the composite system.

\section{Conclusions}

Carbon nanotubes modified by $\mathrm{CF}_{4}$ plasma can reinforce the fluoro elastomer matrix to yield the highest increase in modulus due to better dispersion and enhanced chemical compatibility by introducing electron-rich fluorine atoms. However, for electrical properties, carbon nanotubes modified by mixed acid are predominant in the polymer matrix by removing the amorphous carbon effectively, which results in lower percolation threshold and higher conductivity. This difference in the mechanical and electrical properties would be understood in selecting suitable surface modification.

\section{Acknowledgment}

This work was financially supported by the Special Foundation of CAEP (no. 2008B0302029).

\section{References}

[1] S. Iijima, "Helical microtubules of graphitic carbon," Nature, vol. 354 , no. 6348 , pp. 56-58, 1991.

[2] M. Dresselhaus. and Ph. Avouris, Carbon Nanotubes: Synthesis, Properties and Application, Springer, Berlin, Germany, 2001.

[3] W. Tang, M. H. Santare, and S. G. Advani, "Melt processing and mechanical property characterization of multi-walled carbon nanotube/high density polyethylene (MWNT/HDPE) composite films," Carbon, vol. 41, no. 14, pp. 2779-2785, 2003.

[4] M. L. Shofner, V. N. Khabashesku, and E. V. Barrera, "Processing and mechanical properties of fluorinated single-wall carbon nanotube-polyethylene composites," Chemistry of Materials, vol. 18, no. 4, pp. 906-913, 2006.

[5] M. Moniruzzaman and K. I. Winey, "Polymer nanocomposites containing carbon nanotubes," Macromolecules, vol. 39, no. 16, pp. 5194-5205, 2006.

[6] R. Andrews and M. C. Weisenberger, "Carbon nanotube polymer composites," Current Opinion in Solid State and Materials Science, vol. 8, no. 1, pp. 31-37, 2004.

[7] X.-L. Xie, Y.-W. Mai, and X.-P. Zhou, "Dispersion and alignment of carbon nanotubes in polymer matrix: a review," Materials Science and Engineering R, vol. 49, no. 4, pp. 89-112, 2005.

[8] K. L. Strong, D. P. Anderson, K. Lafdi, and J. N. Kuhn, "Purification process for single-wall carbon nanotubes," Carbon, vol. 41, no. 8, pp. 1477-1488, 2003.

[9] H. G. Chae, T. V. Sreekumar, T. Uchida, and S. Kumar, "A comparison of reinforcement efficiency of various types of carbon nanotubes in polyacrylonitrile fiber," Polymer, vol. 46, no. 24, pp. 10925-10935, 2005.

[10] M. Zhang, L. Su, and L. Mao, "Surfactant functionalization of carbon nanotubes (CNTs) for layer-by-layer assembling of CNT multi-layer films and fabrication of gold nanoparticle/ CNT nanohybrid," Carbon, vol. 44, no. 2, pp. 276-283, 2006.

[11] M. Abdalla, D. Dean, D. Adibempe, E. Nyairo, P. Robinson, and G. Thompson, "The effect of interfacial chemistry on molecular mobility and morphology of multiwalled carbon nanotubes epoxy nanocomposite," Polymer, vol. 48, no. 19, pp. 5662-5670, 2007.

[12] J. A. Kim, D. G. Seong, T. J. Kang, and J. R. Youn, "Effects of surface modification on rheological and mechanical properties of CNT/epoxy composites," Carbon, vol. 44, no. 10, pp. 1898-1905, 2006.

[13] J. Zhu, J. Kim, H. Peng, J. L. Margrave, V. N. Khabashesku, and E. V. Barrera, "Improving the dispersion and integration of single-walled carbon nanotubes in epoxy composites through functionalization," Nano Letters, vol. 3, no. 8, pp. 1107-1113, 2003.

[14] E. T. Thostenson and T.-W. Chou, "Aligned multi-walled carbon nanotube-reinforced composites: processing and mechanical characterization," Journal of Physics D, vol. 35, no. 16, pp. L77-L80, 2002.

[15] B. Yan, K. Qian, Y. Zhang, and D. Xu, "Effects of argon plasma treating on surface morphology and gas ionization property of carbon nanotubes," Physica E, vol. 28, no. 1, pp. 88-92, 2005.

[16] K. S. Ahn, J. S. Kim, C. O. Kim, and J. P. Hong, "Non-reactive rf treatment of multiwall carbon nanotube with inert argon plasma for enhanced field emission," Carbon, vol. 41, no. 13, pp. 2481-2485, 2003.

[17] K. Yu, Z. Zhu, M. Xu, Q. Li, and W. Lu, "Electron field emission from soluble carbon nanotube films treated by hydrogen plasma," Chemical Physics Letters, vol. 373, no. 1-2, pp. 109114, 2003. 
[18] K. Yu, Z. Zhu, Y. Zhang et al., "Change of surface morphology and field emission property of carbon nanotube films treated using a hydrogen plasma," Applied Surface Science, vol. 225, no. 1-4, pp. 380-388, 2004.

[19] W. Chen and X. Tao, "Production and characterization of polymer nanocomposite with aligned single wall carbon nanotubes," Applied Surface Science, vol. 252, no. 10, pp. 3547-3552, 2006.

[20] L. Valentini, D. Puglia, I. Armentano, and J. M. Kenny, "Sidewall functionalization of single-walled carbon nanotubes through $\mathrm{CF}_{4}$ plasma treatment and subsequent reaction with aliphatic amines," Chemical Physics Letters, vol. 403, no. 4-6, pp. 385-389, 2005.

[21] Y. W. Zhu, F. C. Cheong, T. Yu et al., "Effects of $\mathrm{CF}_{4}$ plasma on the field emission properties of aligned multi-wall carbon nanotube films," Carbon, vol. 43, no. 2, pp. 395-400, 2005.

[22] T. Xu, J. Yang, J. Liu, and Q. Fu, "Surface modification of multi-walled carbon nanotubes by $\mathrm{O} 2$ plasma," Applied Surface Science, vol. 253, no. 22, pp. 8945-8951, 2007.

[23] T. Xu, J. Yang, J. Liu, and Q. Fu, "CF${ }^{4}$ plasma-induced grafting of fluoropolymer onto multi-walled carbon nanotube powder," Applied Physics A, vol. 90, no. 3, pp. 431-435, 2008.

[24] C. Vix-Guterl, M. Couzi, J. Dentzer, M. Trinquecoste, and P. Delhaes, "Surface characterizations of carbon multiwall nanotubes: comparison between surface active sites and Raman spectroscopy," Journal of Physical Chemistry B, vol. 108, no. 50, pp. 19361-19367, 2004.

[25] L. Vaisman, G. Marom, and H. D. Wagner, "Dispersions of surface-modified carbon nanotubes in water-soluble and water-insoluble polymers," Advanced Functional Materials, vol. 16, no. 3, pp. 357-363, 2006.

[26] Q. Zhang, S. Rastogi, D. Chen, D. Lippits, and P. J. Lemstra, "Low percolation threshold in single-walled carbon nanotube/ high density polyethylene composites prepared by melt processing technique," Carbon, vol. 44, no. 4, pp. 778-785, 2006. 

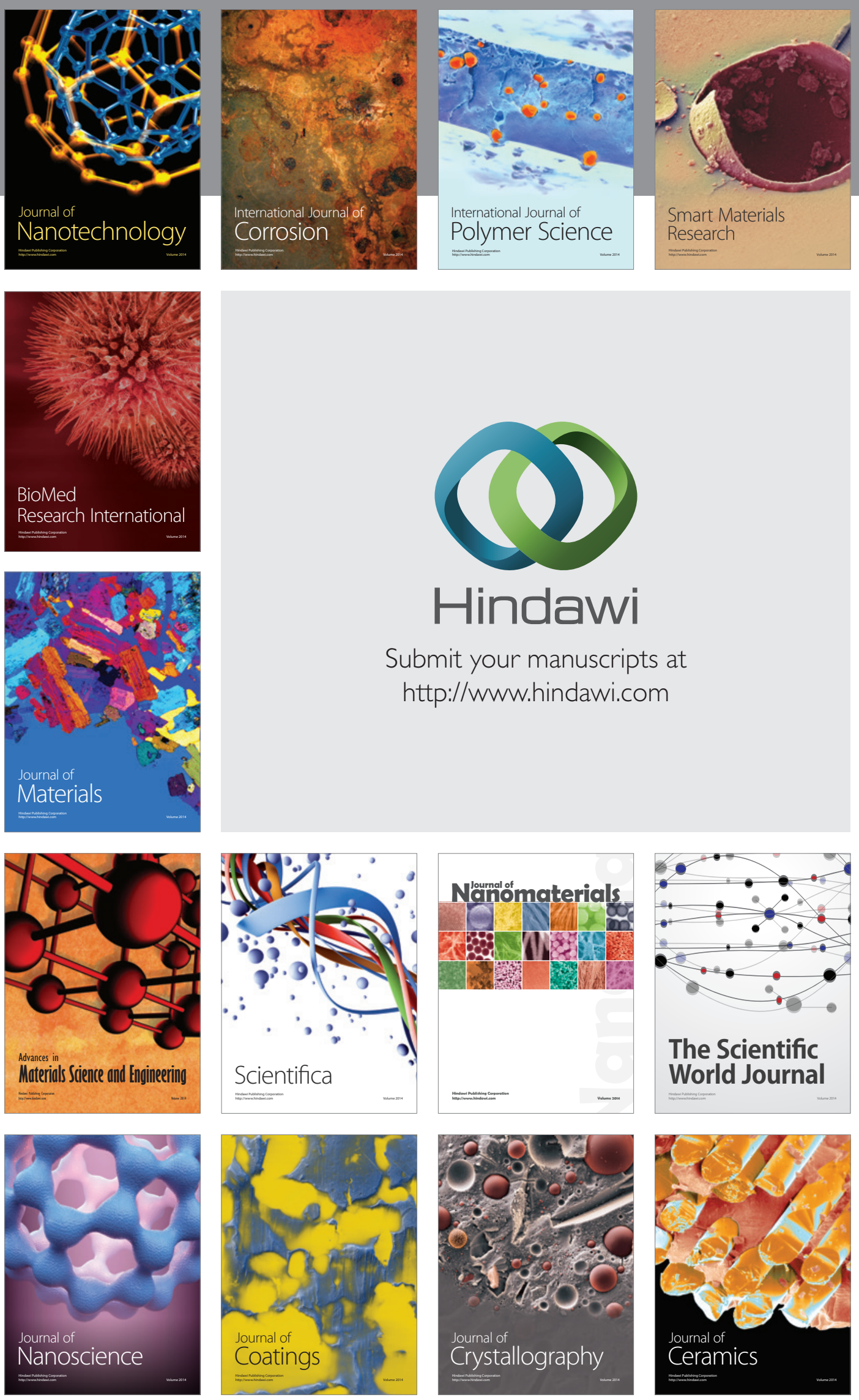

The Scientific World Journal

Submit your manuscripts at

http://www.hindawi.com

\section{World Journal}

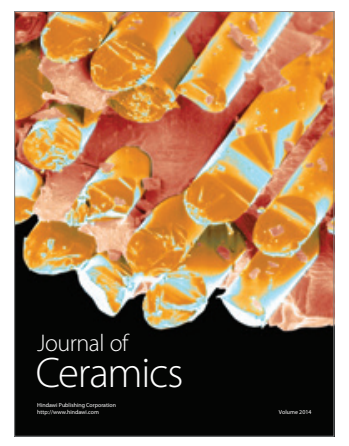

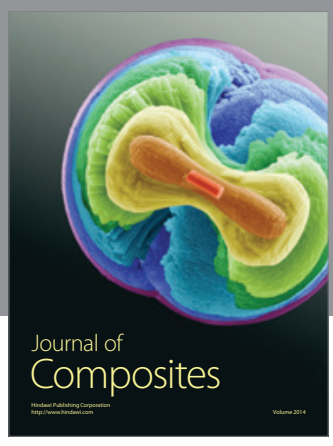
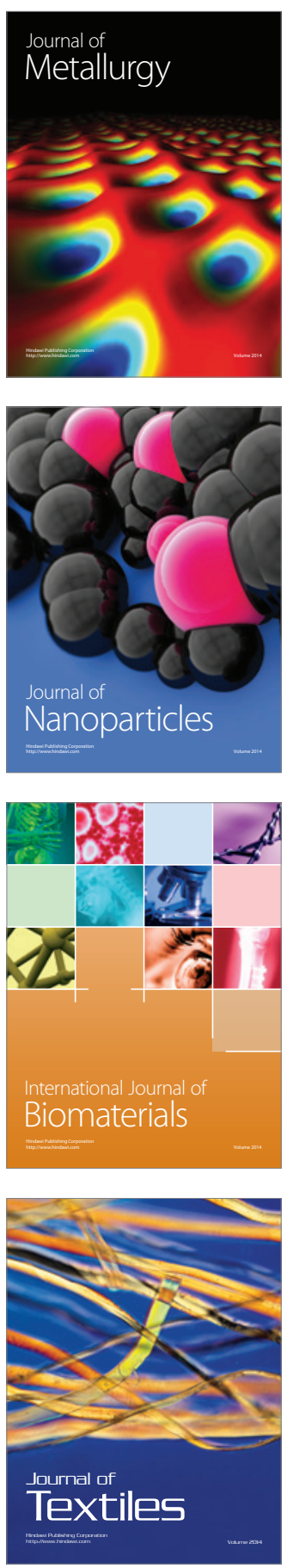